Western University

Scholarship@Western

Physics and Astronomy Publications

Physics and Astronomy Department

2013

\title{
A Biosensor based on Periodic Arrays of Gold Nanodisks under Normal Transmission
}

\author{
Hao Jiang \\ Western University \\ Tingjie Li \\ Western University \\ Erden Ertorer \\ Western University \\ Jun Yang \\ Western University \\ Jayshri Sabarinathan \\ Western University \\ See next page for additional authors
}

Follow this and additional works at: https://ir.lib.uwo.ca/physicspub

Part of the Astrophysics and Astronomy Commons, and the Physics Commons

Citation of this paper:

Jiang, Hao; Li, Tingjie; Ertorer, Erden; Yang, Jun; Sabarinathan, Jayshri; and Mittler, Silvia, "A Biosensor based on Periodic Arrays of Gold Nanodisks under Normal Transmission" (2013). Physics and Astronomy Publications. 47.

https://ir.lib.uwo.ca/physicspub/47 
Authors

Hao Jiang, Tingjie Li, Erden Ertorer, Jun Yang, Jayshri Sabarinathan, and Silvia Mittler 
Sensors and Actuators A 189 (2013) 474-480

\section{A Biosensor based on Periodic Arrays of Gold Nanodisks under Normal Transmission}

\section{Hao Jiang, Tingjie Li, Erden Ertorer, Jun Yang, Jayshri Sabarinathan and Silvia Mittler*}

Hao Jiang, Department of Electrical and Computer Engineering, the University of Western Ontario, London, ON N6A 5B9, Canada

Tingjie Li, Department of Mechanical and Materials Engineering, the University of Western Ontario, London, ON N6A 5B9, Canada

Erden Ertorer, Department of Physics and Astronomy, the University of Western Ontario, London, ON N6A 5B9, Canada

Jun Yang, Department of Mechanical and Materials Engineering, the University of Western Ontario, London, ON N6A 5B9, Canada

Jayshri Sabarinathan, Department of Electrical and Computer Engineering, the University of Western Ontario, London, ON N6A 5B9, Canada

Silvia Mittler, Department of Physics and Astronomy, the University of Western Ontario, London, ON N6A 5B9, Canada

Corresponding author: Silvia Mittler, email: smittler@uwo.ca, tel: 519-661-2111 ext. 88592. 


\begin{abstract}
We present a biosensor based on periodic arrays of gold nanodisks patterned on top of a glass substrate. The sensor's resonance wavelength, peak linewidth and figure of merit were studied both in experiments and in simulations. We analyzed the dependence of the sensor's resolution on the inherent figure of merit of the sensor structure and the signal to noise ratio of the detection system. The best achieved refractive index resolution is $1.5 \times 10^{-4} \mathrm{RIU}$ and the detection limit on and antigen-antibody binding is better than $1 \mathrm{ng} / \mathrm{mL}$.
\end{abstract}

\title{
1. Introduction
}

Noble metal nanoparticles have been widely investigated in label-free biological sensor applications due to the localized surface plasmon resonance (LSPR), which is a coherent oscillation of the free electron gas in metal nanoparticles excited by electromagnetic radiation. When molecules bind to the surface of nanoparticles, a shift in the resonance peak position can be detected in absorption spectroscopy [1-8]. Sensors based on LSPR are highly sensitive and label-free, i.e. functioning without tags. LSPR sensors are therefore very useful in studying biomolecular interactions and provide real-time information on binding kinetics and concentrations of the target biomolecules. This information is valuable for applications in medical diagnostics, such as the detection of virus [9] and in cancer diagnostics [10]. The optical detection system used for LSPR sensors can be achieved using common laboratory optical and mechanical components, making the equipment more cost-effective than the conventional surface plasmon resonance sensor.

Improving the limit of detection of the LSPR sensor is the main focus of this field and the essential target is to increase the sensitivity and to reduce the uncertainty in determining the resonance position $[11,12]$. The sensitivity of metal nanoparticles is highly dependent on the resonance wavelength $[13,14]$, especially when the nanoparticles are embedded in a homogeneous medium [13]. For the metal nanoparticles immobilized on a substrate, the sensitivity is found to be significantly affected by the spatial overlap between the resonance mode and the substrate [15-19]. Other plasmon characteristics, such as the field decay length and field confinement, also strongly affect the sensitivity [11]. The detection uncertainty is determined by the measurement system, signal-to-noise $(\mathrm{S} / \mathrm{N})$ ratio of the spectrum and the spectral linewidth [11, 12]. Among these factors, the spectral linewidth is a directly inherent property of the metal nanoparticles being used. To reduce the detection uncertainty, a sharp spectral line shape (defined by full width at half maximum (fwhm)) is favoured. 
Considering the spectral linewidth, single nanoparticles $[4,6,18,20]$ are more advantageous than a randomly-interacting ensemble with inhomogeneous broadening effects. For coherently-interacting nanoparticle arrays, however, the linewidth can be significantly reduced owing to the coherent interaction of the nanoparticles in a periodic array, suggesting that a periodic array can perform better than single nanoparticles in LSPR sensing [21]. Another significant advantage of using periodic arrays is that, metal nanoparticles can be closely-packed into a very high density without broadening the extinction peak line shape, which can increase the strength of the extinction or scattering signal and thus improve the sensor's $\mathrm{S} / \mathrm{N}$ ratio. In addition, the periodic array can tune the resonance over a wide range of wavelengths, very convenient for optimizing the sensor performance. Different periodic arrays with well separated LSPR spectrum ranges may open new routes towards multiplexed detection.

In this work, we demonstrate a biosensor based on periodic arrays of gold nanodisks. The sensor is schematically depicted in Figure 1. The array of gold nanodisks is patterned into a 2-D square lattice periodic array on top of a BK7 glass substrate. The diameter of the nanodisk $d$ is around $230 \mathrm{~nm}$, the height $h$ is around $50 \mathrm{~nm}$ and the lattice constant $a$ (also called periodicity, center-to-center particle spacing) varies from $400 \mathrm{~nm}$ to $650 \mathrm{~nm}$ with incremental steps of $50 \mathrm{~nm}$. The extinction spectrum of the sensor structure is measured by a transmission setup.

These design parameters of the periodic arrays were chosen by considering the fabrication resolution, the plasmon peak locations and the wavelength of the grating order. These parameters were simulated to demonstrate strong effects of coherent interactions on the particle plasmon resonance. We weree focused on studying the major sensing features of the periodic arrays, including detection uncertainty, figure of merit and the limit of detection. The discovered trends can provide guidance to push the sensor's performance to be comparable with commercial sensor system. In this paper, first the sensing characteristics will be presented and discussed. Then an application in detecting antibody-antigen recognition will be demonstrated.

\section{Experimental method}

\subsection{Sample fabrication}

The gold nanodisk arrays in square lattice were fabricated on BK7 glass substrates (Fisher Scientific Inc.) by electron beam lithography (EBL) [21]. The fabrication process is schematically depicted in Figure 2. After the BK7 substrate was thoroughly cleaned 
using nanostrip (Cyantek Inc.), $300 \mathrm{~nm}$ thick photoresist (ZEP520A, ZEON Corporation) was first spin-coated on top. A $2 \mathrm{~nm}$ thick sacrificial chromium layer was then evaporated on top of the photoresist to make the substrate sufficiently conductive. Pattern of nanodisk arrays, accurately controlled by the EBL system, were then exposed onto the photoresist. After EBL, the chromium layer was first removed by a chromium etchant $(\mathrm{Cr}$ etchant 1020, Transene Company Inc.) followed by developing the photoresist in amyl acetate (Sigma-Aldrich) for 180 seconds. After developing the photoresist, $2 \mathrm{~nm}$ chromium and $50 \mathrm{~nm}$ gold were sequentially deposited on the patterned photoresist by electron beam evaporation deposition. (SEM images are provided in the supplementary information section.) Finally a lift-off process of the sample immersed into N-methyl-2pyrrolidone solvent removed the photoresist together with the metal on its top, leaving the periodic array of gold nanodisks on the BK7 substrate. The fabricated gold nanodisks are in circular shape with diameters equal to $230 \mathrm{~nm}$ and a height of $50 \mathrm{~nm}$.

The fabricated sensor device was integrated with a transparent flow cell for conveniently introducing chemical solutions into the device. The flow cell was constructed by sealing the sensor device with a cover glass (soda-lime microscope slide) on top, in a similar configuration described by Weisser et al. [22]. As demonstrated in Figure 3, glasses were used on both sides for their excellent optical quality in terms of transmission in the nearinfrared spectral regime. In comparison, the widely used PDMS fluidic channels are not flat enough which produces a severe interference pattern in the measured extinction spectra. The sensor device and the cover glass were sealed by a $500 \mu \mathrm{m}$ thick silicone isolator film between them. The soft silicone film, with predefined fluidic channel pattern, seals the two glasses by applying a mild mechanical pressure. Ports were mechanically drilled through the cover glass as inlets and outlets for the fluids. In order to seal each port, one piece of PDMS film was permanently bonded to the cover glass at an elevated temperature following an UV ozone treatment. The steel injection tubes were then inserted through the PDMS, through the ports and into the fluidic channel. This method provides a tightly sealed fluidic channel for the sensor device, with great optical transparency in the near-infrared. In addition, since no glue was involved in the construction of the flow cell, the possibility of the diffusing glue components contaminating the sensor surface was completely avoided.

\subsection{Experimental setup}

The extinction spectra of the sensor were measured by the transmission of linearly polarized (in the direction along an axis of periodicity) broad-band light focused by a $10 \times$ objective $(\mathrm{NA}=0.25)$ onto the device surface positioned perpendicular to the beam. The beam size to interrogate the device is around $40 \mu \mathrm{m} \times 40 \mu \mathrm{m}$, which is sufficiently smaller 
than the size of the fabricated array in order to eliminate edge effects. The transmitted light was collected by a $20 \mathrm{x}$ objective (NA $=0.2$, working distance $=20 \mathrm{~mm}$ ), coupled into a spectrometer (HR2000, Ocean Optics, 2048 pixels). The extinction spectrum was calculated as $\operatorname{Ext}(\lambda)=1-\mathrm{I}_{\operatorname{dev}}(\lambda) / \mathrm{I}_{\text {ref }}(\lambda)$, with $\mathrm{I}_{\operatorname{dev}}(\lambda)$ being the light transmitted through the nanoparticles and $\operatorname{Iref}_{\mathrm{ref}}(\lambda)$ being the reference spectrum.

\section{Sensing characteristics}

\subsection{Peak and linewidth}

The measured spectra of the devices immersed in de-ionized water (Milli Q, Milli-Pore) are plotted in Figure 4. The measured spectra show that the dipolar resonance of the gold nanodisks is strongly dependent on the lattice constant. The LSPR peak wavelength and linewidth fwhm are plotted versus the lattice constant in Figure 5(a) and (b). Also plotted are the results from 3-D finite difference time domain (FDTD) simulations using the configuration described elsewhere [21,23].

As the lattice constant increases, the LSPR peak red-shifts and the linewidth narrows significantly. In the experiments, as the $a$ increases from $400 \mathrm{~nm}$ into $650 \mathrm{~nm}$, the LSPR peak shifts from $801 \mathrm{~nm}$ into $1011 \mathrm{~nm}$ while the fwhm decreases from $270 \mathrm{~nm}$ to $72 \mathrm{~nm}$. The dependence of the plasmon peak of the periodic array on the lattice constants has been investigated by many researchers. Meier et al. have theoretically studied the dipolar interactions of periodic arrays of nanoparticles and predicted array effects on the plasmon peak and the radiative damping [24], which have been observed in many experiments on 2D periodic arrays of metal nanoparticles [25-28]. The trend of the peak wavelength and linewidth changes is determined by the coupling of the periodic gold nanoparticles array through the grating order at a grazing angle [24, 25]. At a given wavelength, the condition for the emergence of the radiative grating order is described by the critical grating constant $a_{c}$, meaning that for $a<a_{c}$ the grating order is evanescent and for $a>a_{c}$ the grating order is radiative [24, 25]. For the $1^{\text {st }}$ grating order propagating in the substrate at a grazing angle, the wavelength corresponding to the critical grating constant is given by $\lambda=n_{\text {sub }} \times a_{c}$, where $n_{\text {sub }}=1.515$, the refractive index of the glass substrate. This relation is plotted as the solid line in Figure 5(a) to help determining whether the substrate grating order is evanescent or radiative. For the range of lattice constants studied in this work, $a$ is always smaller than $a_{c}$ meaning that the grating order is always of evanescent nature. We are focused on the evanescent grating order range because this range can produce narrow extinction peak, which can reduce the detection uncertainty as will be discussed later in the article. 


\subsection{Detection uncertainty}

Since the sensor's function is based on measuring the amount of peak shift vs. change of refractive index, the uncertainty in detecting the peak shift plays a dominat role on the sensor's resolution. The detection uncertainty is determined by the algorithm used to quantify the peak shift, the signal-to-noise $(\mathrm{S} / \mathrm{N})$ ratio of the measurement system and the sensor structure's extinction spectrum linewidth.

To quantify the peak shift, we followed the algorithm described by Dahlin et al. [29] to calculate the centroid peak, $\lambda_{c}$, of each spectrum. This method has been successfully implemented by Chen et al. [10] to achieve a remarkable detection uncertainty of $9.9 \times 10^{-}$ ${ }^{5} \mathrm{~nm}$. The details of calculations are illustrated in the supplementary information section. The detection uncertainty $\lambda_{u n}$ in quantifying the peak location for each array was estimated from the statistical standard deviation of $\lambda_{c}$ from all the spectra recorded over 10 minutes.

The $\mathrm{S} / \mathrm{N}$ ratio of the measurement system is mainly determined by the light source intensity spectrum, optical lens and filters and the spectrometer's sensitivity and is therefore wavelength-dependent. The $S / N$ ratio is given by $S(\lambda) / \sigma(\lambda)$, where $S(\lambda)$ is the intensity spectrum and $\sigma(\lambda)$ is the statistical standard deviation in the intensity spectrum. The $\mathrm{S} / \mathrm{N}$ ratio of the entire spectrum range was plotted in the supplementary information section. Different array configuration has a peak in a different wavelength, and therefore was measured using a different $\mathrm{S} / \mathrm{N}$ ratio. The $\mathrm{S} / \mathrm{N}$ ratio of the measurement system used by each array is plotted as red circles in Figure 6 . As the lattice constant increases, the peak red-shifts and the $\mathrm{S} / \mathrm{N}$ ratio decreases because the silicon-based detector array of the spectrometer has a decreasing sensitivity in the spectrum range above $750 \mathrm{~nm}$.

For each array, the peak linewidth and detection uncertainty are also plotted together in Figure 6. The trend of the uncertainty is first decreasing and then increasing, with a minimum at $a=550 \mathrm{~nm}$ and the best detection uncertainty is around $0.012 \mathrm{~nm}$. Such a trend is caused by the effects of $\mathrm{S} / \mathrm{N}$ ratio and the peak linewidth together. In order to understand their effects, we numerically calculated the detection uncertainty of noisy spectra with a given peak linewidth and $\mathrm{S} / \mathrm{N}$ ratio (the details of the calculations are demonstrated in the supplementary information section). Figure 7 shows the calculated detection uncertainty vs. linewidth fwhm and the $\mathrm{S} / \mathrm{N}$ ratio. The calculation predicts the uncertainty to be $0.015 \mathrm{~nm}$ for the device $a=550 \mathrm{~nm}$, which is close to the experimental value. For any given $\mathrm{S} / \mathrm{N}$ ratio, a sharper peak delivers a smaller uncertainty, because the centroid peak shift can be quantified more accurately in a sharper line shape. For a given

peak linewidth, as the $\mathrm{S} / \mathrm{N}$ ratio increases, the uncertainty is significantly reduced. This analysis could qualitatively explain the experimental trend of the uncertainty. The S/N ratio and the peak linewidth for the array are taking opposite effects. The increased 
detection uncertainty for small lattice constants is due to the broadened peak and the increased detection uncertainty for large lattice constants is due to the low $\mathrm{S} / \mathrm{N}$ ratio.

\subsection{Refractive index sensitivity and resolution}

The bulk refractive index sensitivity $m_{B}$ is defined as $m_{B}=\delta \lambda_{L S P R} / \delta n_{B}$ where $n_{B}$ is the background refractive index of the solvent. In order to measure $m_{B}$, water and isopropanol (IPA) mixtures in different ratios were injected onto the sensor to induce a change of $n_{B}$. The extinction peak of each solvent refractive index was measured and the sensitivity was determined from the slope of the linear fit of $\lambda_{L S P R}$ vs. $n_{B}$. The refractive index (RI) resolution is defined as $n_{B}=3 \lambda_{u n} / m_{B}$ and plotted together with the detection uncertainty in Figure 6. This definition is related to the confidence level of $99.7 \%$ in determining the peak shift [31]. It can be seen that, the RI resolution in this case is mainly governed by the detection uncertainty, because the change in the sensitivity is much less significant. The best RI resolution is $<1.5 \times 10^{-4}$ refractive index unit (RIU), which is obtained from the lattice configuration of $a=550 \mathrm{~nm}$. Therefore, in the given measurement system, the optimized sensor array has a lattice constant of $550 \mathrm{~nm}$.

\subsection{Figure of merit of the periodic array}

The refractive index resolution is determined by the nanoplasmonic structure and the $\mathrm{S} / \mathrm{N}$ ratio of the detection system together. If we only consider the inherent properties of the sensor structure, i.e., if we assume the $\mathrm{S} / \mathrm{N}$ ratio is the same for all wavelength range, the performance of the sensor structure can be well described by the figure of merit defined as $\mathrm{FOM}_{\mathrm{B}}=m_{B} /$ fwhm. $m_{B}$ and fwhm can be in either energy units or wavelength units and the calculated values using different units are approximately equivalent [30]. At a given change of refractive index, a higher sensitivity produces a larger peak shift, and a sharper peak reduces the detection uncertainty. Therefore, a higher figure of merit can achieve a better refractive index resolution. The figure of merit can thus directly evaluate the performance of the sensor structure, excluding the effects of the $\mathrm{S} / \mathrm{N}$ ratio of the measurement system. The measured $\mathrm{FOM}_{\mathrm{B}}$ are plotted against the lattice constant in Figure 5(c). The bulk refractive index figure of merit can increase from 0.9 into 3.8: an enhancement of nearly half an order of magnitude. This improvement is mainly obtained from the sharpened extinction peak due to the coherent interactions in the periodic array [21].

The figure of merit suggests that the structure with $a=650 \mathrm{~nm}$ has the best sensing potential, however, its performance in experiments was compromised by the low $\mathrm{S} / \mathrm{N}$ 
ratio in this spectrum range. In order to improve the RI resolution of an LSPR sensor equipment, the nanoplasmonic structure with high figure of merit should be equipped with a matched detection system providing high $\mathrm{S} / \mathrm{N}$ ratio in the corresponding spectrum range.

\subsection{Antibody-antigen recognition}

For sensing applications, we chose the lattice configuration with the best RI resolution, i.e. $a=550 \mathrm{~nm}$. We implemented this sensor to detect the binding of an antigen onto the antibody immobilized on the sensor surface. The chosen antibody is anti-human IgG (Sigma Aldrich) and the antigen is human IgG (Sigma Aldrich). Such a study provides a proof of concept for applying this sensor in medical diagnostics, such as early detection of leukemia and lung cancer.

We first implemented the biotin-streptavidin interaction to immobilize the antibody on the surface of gold. The basic strategy is to first immobilize streptavidin on the surface of gold and then introduce the biotinylated antibody which binds to streptavidin. To immobilize the streptavidin, we follow the procedures described by Choi et al. [32]. The procedures are schematically shown in Figure 8(a) and the corresponding sensor response for the entire surface functionalization procedures is given in Figure 8(b). The extinction spectrum was acquired every 15 seconds. After the sample $(a=550 \mathrm{~nm})$ was thoroughly cleaned using nanostrip (Cyantek Inc.) and mounted with the flow cell, a mixture of 3mercaptopropanol (3-MPOH) and 11-mercaptoundecanoic acid (11-MUA) in absolute ethanol was continuously flown on the sensor surface at $1 \mu \mathrm{L} / \mathrm{min}$ for 18 hours to form a well organized self-assembled monolayer (SAM) on the surface of the gold nanodisks with carboxylic and hydroxyl terminal groups. The flow rate was controlled by a syringe pump (Braintree Scientific Inc.) The molar ratio of 3-MPOH and 11-MUA is 10:1, and the total concentration is $10 \mathrm{mM}$. Then ethanol followed by de-ionized water was flown at $1 \mathrm{~mL} / \mathrm{min}$ for 30 minutes to thoroughly rinse the surface. A 1:1 mixture of $0.2 \mathrm{M} 1$ ethyl-3-(3-dimethylaminopropyl) carbodiimide (EDC) and 0.4 M N-hydroxysuccinimide (NHS) solutions was then flown onto the sensor at $10 \mu \mathrm{L} / \mathrm{min}$ for 3 hours to activate the terminal carboxylic groups into amine-reactive succinimide esters. Then streptavidin (200 $\mu \mathrm{g} / \mathrm{mL}$ ) (Sigma-Aldrich) in PBS buffer $(\mathrm{pH}=7.4)$ (Sigma-Aldrich) was injected at a rate of $10 \mu \mathrm{L} / \mathrm{min}$ for 25 minutes and bonded to the activated carboxylic terminal groups of the mixed SAM. This procedure was repeated once to enhance the immobilization. After rinsing the sensor with PBS buffer, $10 \mu \mathrm{g} / \mathrm{mL}$ bovine serum albumin (BSA) (SigmaAldrich) in PBS buffer was flown at $10 \mu \mathrm{L} / \mathrm{min}$ for 30 minutes, in order to block the nonreacted sites. As observed in Figure 8(b), the peak red-shifts due to the binding of the streptavidin and BSA to the surface of the gold nanodisks. In the following, biotinylated 
anti-human $\operatorname{IgG}(10 \mu \mathrm{g} / \mathrm{mL})$ in PBS buffer is flown onto the sensor at $10 \mu \mathrm{L} / \mathrm{min}$ for more than one hour. The biotinylated antibody binds to streptavidin and a saturated redshift of around $0.7 \mathrm{~nm}$ was acquired in the end. This procedure was repeated once to enhance the immobilization. Then the sensor was thoroughly rinsed with PBS solution and a stable baseline signal was acquired.

To sense biomolecular recognition, human $\operatorname{IgG}$ of different concentrations $(1 \mathrm{ng} / \mathrm{mL}-$ $100 \mu \mathrm{g} / \mathrm{mL}$ ) were sequentially tested in an order of increasing concentration. Each concentration of human $\operatorname{IgG}$ was continuously flown onto the sensor at $10 \mu \mathrm{L} / \mathrm{min}$ for 15 minutes. The human IgG biomolecules bound to the anti-human IgG and gradually reached equilibrium. Then PBS was injected to rinse of the non-specific bound molecules and the sensor signal corresponding to each concentration was reported from the spectra acquired 1 minute after rinsing. After the sensor was rinsed for 15 minutes, a higher concentration of human IgG was injected and the same detection scheme was carried out repeatedly. The sensor response vs. concentration was plotted in Figure 9. The peak shift above the red line can be trusted to be a real detectable shift. It can be determined from this graph that the limit of detection is better than $1 \mathrm{ng} / \mathrm{mL}$.

\section{Discussions}

Although the sensor can basically function, the peak shift is not as large as expected. A simple simulation showed that, upon full coverage of the antigen biomolecules, at least 3 $\mathrm{nm}$ peak shift should be obtained. In our experiment, the maximum peak shift is less than $1 \mathrm{~nm}$. The reasons for this were the low quality of the implemented surface chemistry, which could not reveal the sensor's full potential. In addition, the SAM layer on the surface of gold nanodisks is not robust enough, which can be easily destroyed when a strong basic condition was introduced for the purpose of dissociating the antibodyantigen binding. Improvement on the surface chemistry is necessary, and a possible approach might be an implementation of a thin polyethylene glycol (PEG) based SAM layer [33].

The measurement system used by Chen et al. [10] can reach a detection uncertainty of $9.9 \times 10^{-5} \mathrm{~nm}$, approximately $1 \times 10^{-4} \mathrm{~nm}$. In our experiment, the minimum uncertainty is $0.012 \mathrm{~nm}$. It should be pointed out, that, the differences are due to the $\mathrm{S} / \mathrm{N}$ ratio of the detection system rather than the inherent properties of the nanoplasmonic structure. The achieved figure of merit of the periodic array is around 3.8, which is an excellent number compared to other gold nanoparticle devices summarized by Mayer et al. [34]. We have further calculated that, to reach the $1 \times 10^{-4} \mathrm{~nm}$ range using the array with highest figure of merit, the required $\mathrm{S} / \mathrm{N}$ ratio of the measurement system needs to be $3.2 \times 10^{4}$ (supplementary information). However, in our current experiments, the maximum $\mathrm{S} / \mathrm{N}$ 
ratio is less than 400 which indicates that the detection system needs improvement. A high intensity and stabilized broadband light source and a more sensitive spectrometer should be implemented and both should be optimized for the array with highest figure of merit. Once the detection uncertainty can reach $1 \times 10^{-4} \mathrm{~nm}$ range, the sensor can achieve a refractive index resolution in the range $1 \times 10^{-6} \mathrm{RIU}$, comparable to the conventional surface plasmon sensor.

\section{Conclusions}

In this paper, we described a biosensor based on periodic arrays of gold nanodisks. The sensor structures with varying lattice constant were fabricated and studied experimentally and in simulations. The important sensing characteristics, including LSPR peak wavelength, peak linewidth fwhm, figure of merit, detection uncertainty and refractive index resolution, were investigated in detail. The effects of signal-to-noise on the sensor's performance were also studied. The highest obtained figure of merit is around 3.8 from the structure with $a=650 \mathrm{~nm}$._The best refractive index resolution $<1.5 \times 10^{-4} \mathrm{RIU}$ was obtained from the structure with $a=550 \mathrm{~nm}$. The structure with $a=550 \mathrm{~nm}$ was used in the biosensing application to detect the binding of human $\operatorname{IgG}$ to the anti-human $\mathrm{IgG}$ antibody. The sensor was able to reach a limit of detection better than $1 \mathrm{ng} / \mathrm{mL}$.

\section{Acknowledgements}

The authors acknowledge the funding from BIOPSYS, a strategic network funded by Natural Sciences and Engineering Research Council of Canada (NSERC). We would also like to acknowledge the funding of TEN program from Canadian Institute for Photonic Innovations (CIPI). We would like to thank Dr. Gilbert Walker and Dr. Weiqing Shi for biochemical procedures. The device fabrication was performed at the University of Western Ontario Nanofabrication Facility. The simulations were carried out using

SHARCNET parallel computation facilities. We would also like to acknowledge the technical support on the 3-D FDTD simulations from Lumerical Solutions Inc. S.M. likes to acknowledge the Canadian Government for the CRC program. 


\section{References}

[1] K. A. Willets and R. P. Van Duyne, "Localized surface plasmon resonance spectroscopy and sensing," Annu. Rev. Phys. Chem., vol. 58, pp. 267-297, 2007.

[2] J. N. Anker, W. P. Hall, O. Lyandres, N. C. Shah, J. Zhao, and R. P. Van Duyne, "Biosensing with plasmonic nanosensors," Nature Materials, vol. 7, pp. 442-453, 2008.

[3] P. Englebienne, "Use of colloidal gold surface plasmon resonance peak shift to infer affinity constants from the interactions between protein antigens and antibodies specific for single or multiple epitopes," the Analyst, vol. 123, pp. 1599-1603, 1998.

[4] A. D. McFarland and R. P. Van Duyne, "Single silver nanoparticles as realtime optical sensors with zeptomole sensitivity," Nano Letters, vol. 3, no. 8, pp. 1057-1062, 2003.

[5] A. J. Haes and R. P. Van Duyne, "A nanoscale optical biosensor: Sensitivity and selectivity of an approach based on the localized surface plasmon resonance spectroscopy of triangular silver nanoparticles," Journal of the American Chemical Society, vol. 124, no. 35 , pp. 10 596-10 604, 2002.

[6] G. Raschke, S. Kowarik, T. Franzl, C. Sonnichsen, T. A. Klar, J. Feldmann, A. Nichtl, and K. Kurzinger, "Biomolecular recognition based on single gold nanoparticle light scattering," Nano Letters, vol. 3, no. 7, pp. 935-938, 2003.

[7] P. Rooney, A. Rezaee, S. Xu, T. Manifar, A. Hassanzadeh, G. Podoprygorina, V. Bohmer, C. Rangan, and S. Mittler, "Control of surface plasmon resonances in dielectrically coated proximate gold nanoparticles immobilized on a substrate," Phys. Rev. $B$, vol. 77, p. 235446, 2008.

[8] A. K. A. Aliganga, I. Lieberwirth, G. Glasser, A.-S. Duwez, Y. Sun, and S. Mittler, "Fabrication of equally oriented pancake shaped gold nanoparticles by SAM templated OMCVD and their optical response," Organic Electronics, vol. 8, no. 2-3, pp. 161 - 174, 2007.

[9] A. A. Yanik, M. Huang, O. Kamohara, A. Artar, T. W. Geisbert, J. H. Connor, and H. Altug, "An optofluidic nanoplasmonic biosensor for direct detection of live viruses from biological media,” Nano Letters, vol. 10, no. 12, pp. 4962-4969, 2010.

[10] S. Chen, M. Svedendahl, M. Kall, L. Gunnarsson, and A. Dmitriev, "Ultrahigh sensitivity made simple: nanoplasmonic label-free biosensing with an extremely low limit-of-detection for bacterial and cancer diagnostics," Nanotechnology, vol. 20, no. 43, p. $434015,2009$.

[11] A. Unger and M. Kreiter, "Analyzing the performance of plasmonic resonators for dielectric sensing," J. Phys. Chem. C, vol. 113, pp. 12 243-12 251, 2009. 
[12] G. J. Nusz, A. C. Curry, S. M. Marinakos, A. Wax, and A. Chilkoti, "Rational selection of gold nanorod geometry for label-free plasmonic biosensors," ACS Nano, vol. 3, no. 4, pp. 795-806, 2009.

[13] M. M. Miller and A. A. Lazarides, "Sensitivity of metal nanoparticle surface plasmon resonance to the dielectric environment," J. Phys. Chem. B, vol. 109, pp. 21556$21565,2005$.

[14] R. Bukasov and J. S. Shumaker-Parry, "Highly tunable infrared extinction properties of gold nanocrescents," Nano Letters, vol. 7, no. 5, pp. 1113-1118, 2007.

[15] A. Dmitriev, C. Hagglund, S. Chen, H. Fredriksson, T. Pakizeh, M. Kall, and D. S. Sutherland, "Enhanced nanoplasmonic optical sensors with reduced substrate effect," Nano Letters, vol. 8, no. 11, pp. 3893-3898, 2008.

[16] E. M. Larsson, J. Alegret, M. Kall, and D. S. Sutherland, "Sensing characteristics of NIR localized surface plasmon resonances in gold nanorings for application as ultrasensitive biosensors," Nano Letters, vol. 7, no. 5, pp. 1256-1263, 2007.

[17] C. Novo, A. M. Funston, I. Pastoriza-Santos, L. M. Liz-Marzan, and P. Mulvaney, "Influence of medium refractive index on the optical properties of single gold triangular prisms on a substrate," J. Phys. Chem. C., vol. 112, no. 1, pp. 3-7, 2008.

[18] L. J. Sherry, S.-H. Chang, G. C. Schatz, R. P. Van Duyne, B. J. Wiley, and Y. Xia, "Localized surface plasmon resonance spectroscopy of single silver nanocubes," Nano Letters, vol. 5, no. 10, pp. 2034-2038, 2005.

[19] W. A. Murray, B. Auguie, and W. L. Barnes, "Sensitivity of localized surface plasmon resonances to bulk and local changes in the optical environment," J. Phys. Chem. C, vol. 113, pp. 5120-5125, 2009.

[20] L. J. Sherry, R. Jin, C. A. Mirkin, G. C. Schatz, and R. P. Van Duyne, "Localized surface plasmon resonance spectroscopy of single silver triangular nanoprisms," Nano Letters, vol. 6, no. 9, pp. 2060-2065, 2006.

[21] H. Jiang J. Sabarinathan, and Silvia Mittler, "Effects of coherent interactions on the sensing characteristics of near-infrared gold nanorings," Journal of Physical Chemistry C, vol. 114, no. 36, pp. 15 243-15 250, 2010 and erratum 2012.

[22] M. Weisser, G. Tovar, S. Mittler-Neher, W. Knoll, F. Brosinger, H. Freimuth, M. Lacher, and W. Ehrfeld, "Specific bio-recognition reactions observed with an integrated Mach-Zehnder interferometer," Biosensors and Bioelectronics, vol. 14, no. 4, pp. 405 411, 1999. 
[23] H. Jiang, J. Sabarinathan, T. Manifar, and S. Mittler, "3-D FDTD analysis of goldnanoparticle-based photonic crystal on slab waveguide," J. Lightwave Technol., vol. 27, no. 13, pp. 2264-2270, 2009.

[24] M. Meier, A. Wokaun, and P. F. Liao, "Enhanced fields on rough surfaces: dipolar interactions among particles of sizes exceeding the Rayleigh limit," J. Opt. Soc. Am. B, vol. 2, no. 6, pp. 931-949, 1985.

[25] B. Lamprecht, G. Schider, R. T. Lechner, H. Ditlbacher, J. R. Krenn, A. Leitner, and F. R. Aussenegg, "Metal nanoparticle gratings: influence of dipoalr particle interaction on the plasmon resonance," Phys. Rev. Lett., vol. 84, no. 20, pp. 4721-4724, 2000.

[26] J. Sung, E. M. Hicks, R. P. Van Duyne, and K. G. Spears, "Nanosphere spectroscopy: dipole coupling in two-dimensional arrays of L-shaped silver nanoparticles," $J$. Phys. Chem. C, vol. 111, pp. 10 368-10 376, 2007.

[27] J. Sung, E. M. Hicks, R. P. Van Duyne, and K. G. Spears, "Nanoparticle spectroscopy: plasmon coupling in finite-sized two-dimensional arrays of cylindrical silver nanoparticles," Journal of Physical Chemistry C, vol. 112, no. 11, pp. 4091-4096, 2008.

[28] C. L. Haynes, A. D. McFarland, L. Zhao, R. P. Van Duyne, G. C. Schatz, L. Gunnarsson, J. Prikulis, B. Kasemo, and M. Kall, "Nanoparticle optics: the importance of radiative dipole coupling in two-dimensional nanoparticle Arrays," J. Phys. Chem. B, vol. 107, pp. 7337-7342, 2003.

[29] A. B. Dahlin, J. O. Tegenfeldt, and F. Hook, "Improving the instrumental resolution of sensors based on localized surface plasmon resonance," Analytical Chemistry, vol. 78, no. 13, pp. 4416-4423, 2006.

[30] M. A. Otte, B. Sepulveda, W. Ni, J. P. Juste, L. M. Liz-Marzan, and L. M. Lechuga, "Identification of the optimal spectral region for plasmonic and nanoplasmonic sensing," ACS Nano, vol. 4, no. 1, pp. 349-357, 2010.

[31] J. Homola and M. Piliarik, "Surface plasmon resonance (SPR) sensors," in Surface Plasmon Resonance Based Sensors, ser. Springer Series on Chemical Sensors and Biosensors, J. Homola and O. S. Wolfbeis, Eds. Springer Berlin Heidelberg, 2006, vol. 4, pp. 45-67.

[32] S. H. Choi, J. W. Lee, and S. J. Sim, "Enhanced performance of a surface plasmon resonance immunosensor for detecting Ab-GAD antibody based on the modified selfassembled monolayers," Biosensors and Bioelectronics, vol. 21, no. 2, pp. 378- 383, 2005. 
[33] J. M. Harris, Poly(ethylene glycol) Chemistry: biotechnical and biomedical applications. Plenum Press, New York, 1992.

[34] K. M. Mayer and J. H. Hafner, "Localized surface plasmon resonance sensors," Chemical Reviews, vol. 111, no. 6, pp. 3828-3857, 2011. 


\section{Figure captions:}

Fig.1 Scheme of the sensor configuration. The geometrical parameters are defined and summarized in the figure.

Fig.2 Fabrication scheme for periodic arrays of gold nanodisks.

Fig.3 Construction of transparent flow cell for transmission measurements in analogy to Weisser et al. [22].

Fig.4 Measured extinction spectra of gold nanodisk arrays with different lattice constants immersed in water. The lattice constant changes from $400 \mathrm{~nm}$ to $650 \mathrm{~nm}$, incremental steps of $50 \mathrm{~nm}$.

Fig.5 Sensing characteristics of the extinction peaks for different lattice constants $a$. (a) The LSPR peak $\lambda_{L S P R}$ vs. $a$. (b) The linewidth fwhm vs. $a$. (c) Figure of merit $\mathrm{FOM}_{\mathrm{B}}$ vs. $a$. The black squares are the measured data while the red circles represent the simulation results.

Fig.6 Experimental detection uncertainty, refractive index resolution, fwhm and $\mathrm{S} / \mathrm{N}$ ratio, for varying lattice constants. In the top figure, fwhm and the $\mathrm{S} / \mathrm{N}$ ratio are plotted, while in the bottom panel, the detection uncertainty and the refractive index resolution are shown in logarithmic scale.

Fig.7 Calculated detection uncertainty vs. linewidth fwhm and the $\mathrm{S} / \mathrm{N}$ ratio.

Fig.8 The surface chemistry procedures for detecting antibody-antigen recognition. a) Schematic of the chemistry procedures. b) Real-time sensor response recorded during various binding steps.

Fig.9 Dose response of human IgG binding to anti-human IgG immobilized on the gold nanodisk surface. The black horizontal line is the base line signal while the green line marks the detection uncertainty. The red line is the decision making threshold which marks the baseline plus three times the detection uncertainty. 
FIG.1

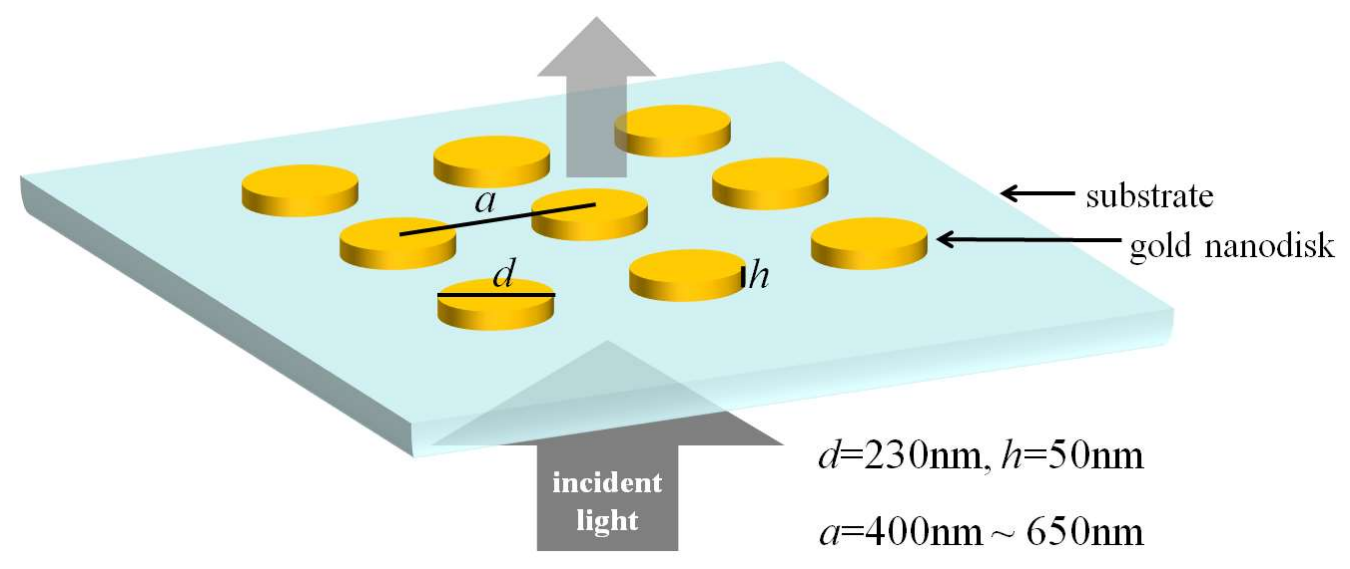

FIG.2

(I)

(II)

(III)

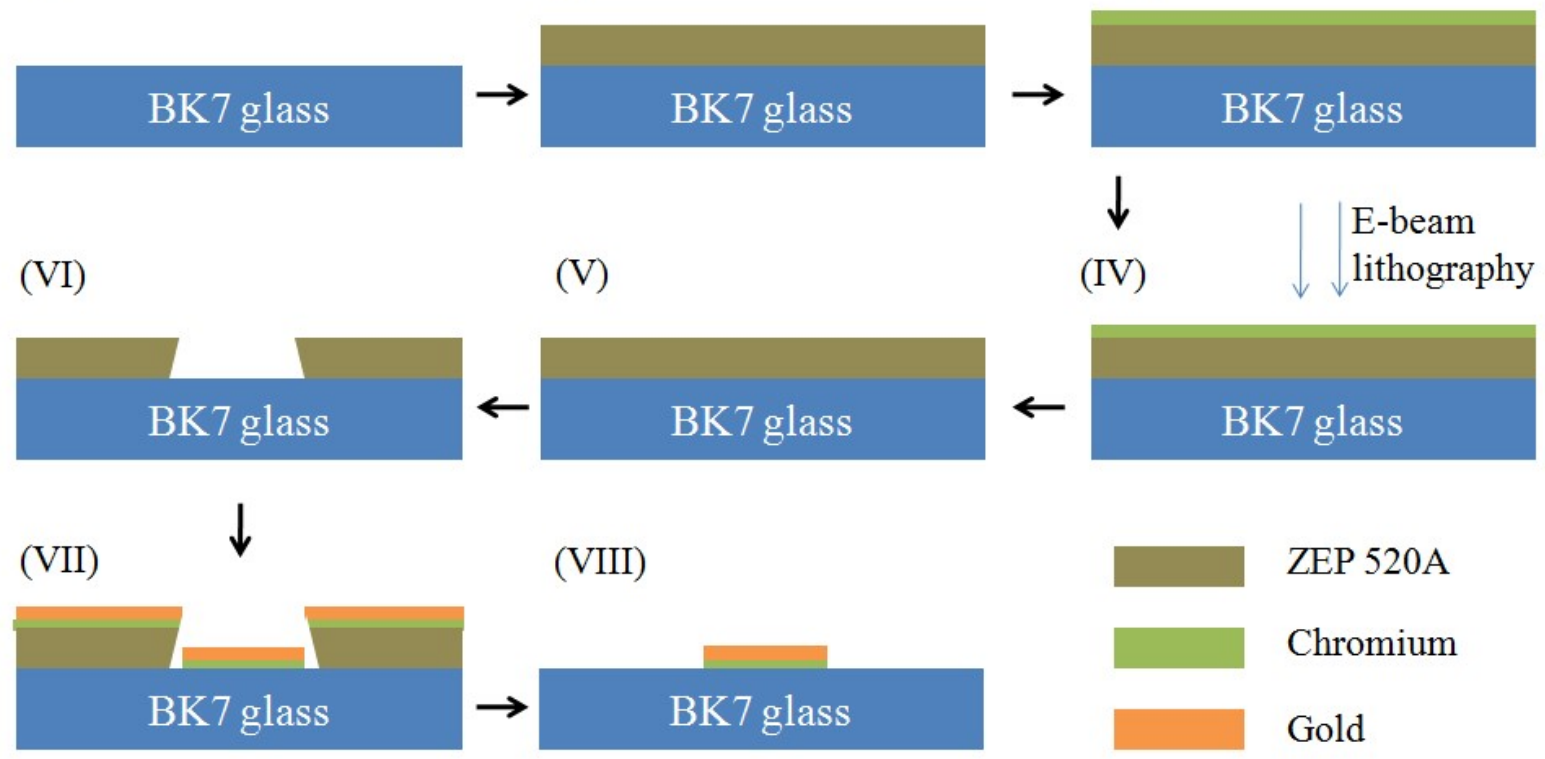


FIG.3

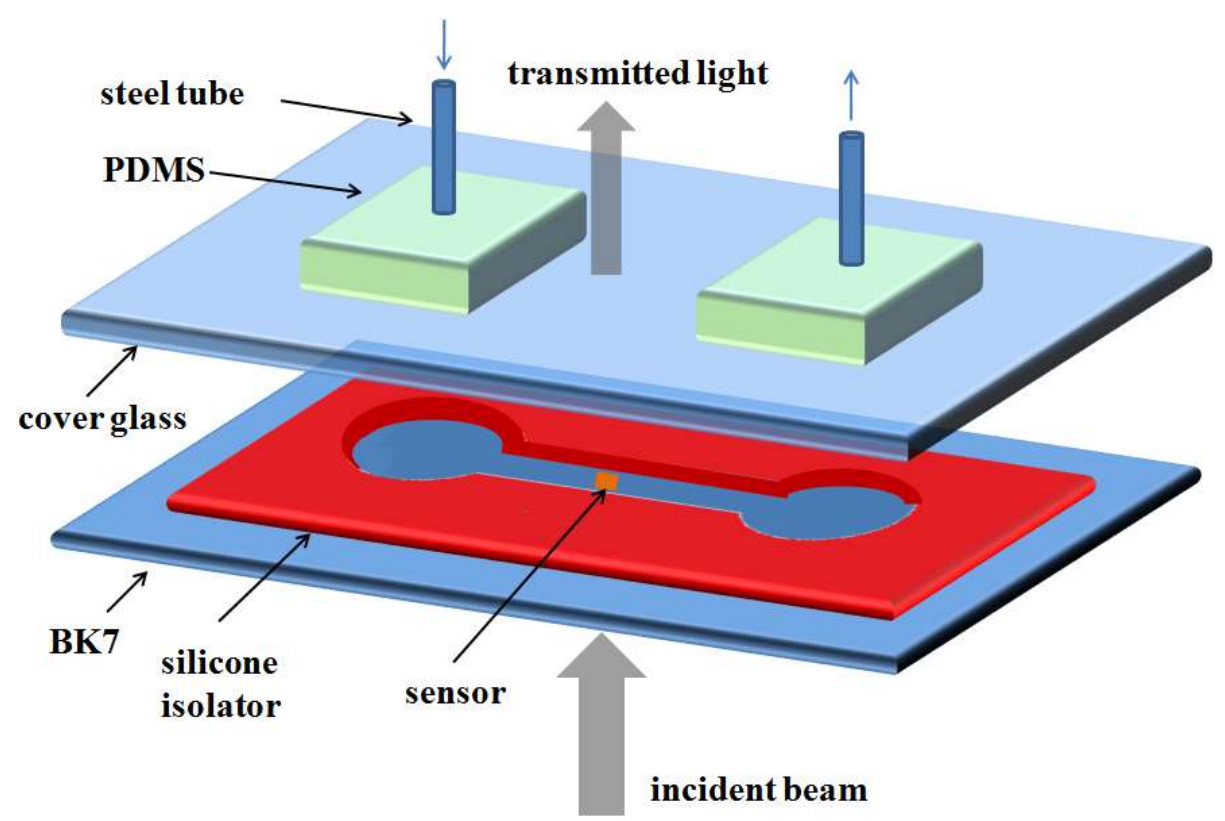

FIG.4

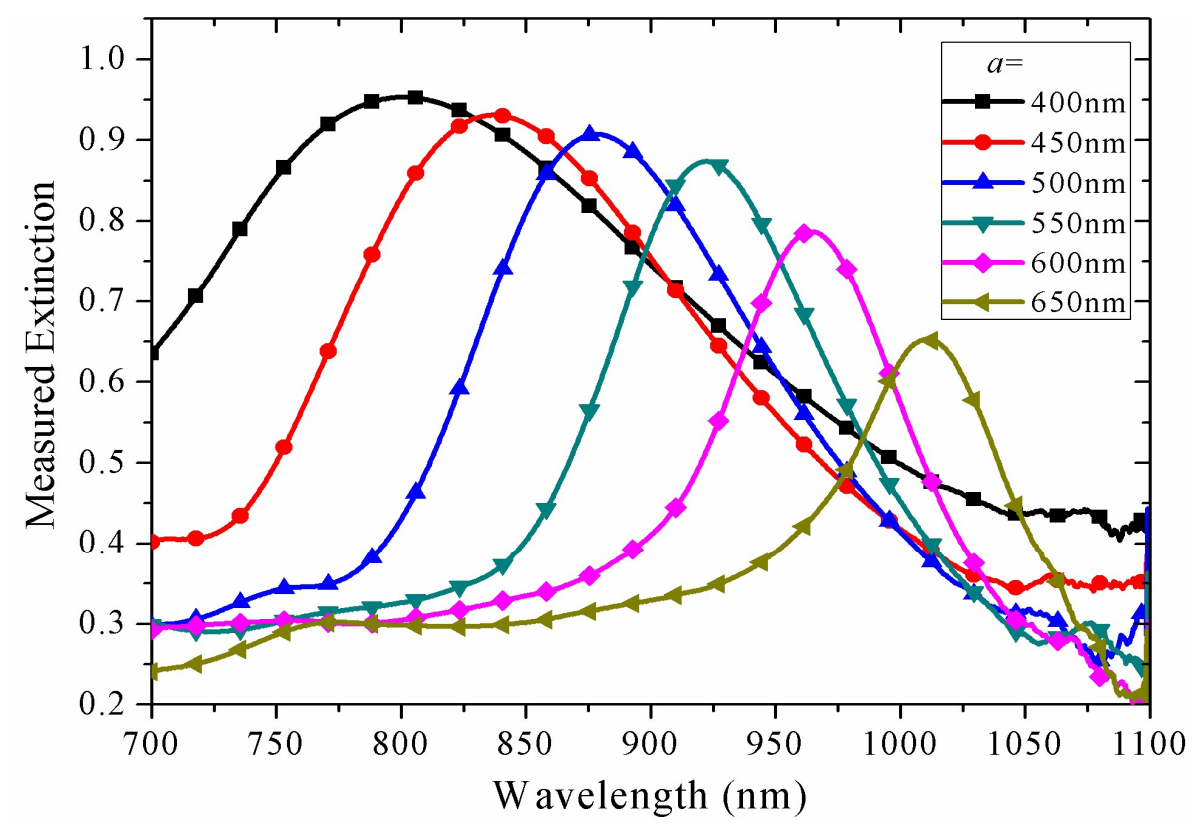




\section{FIG.5a}

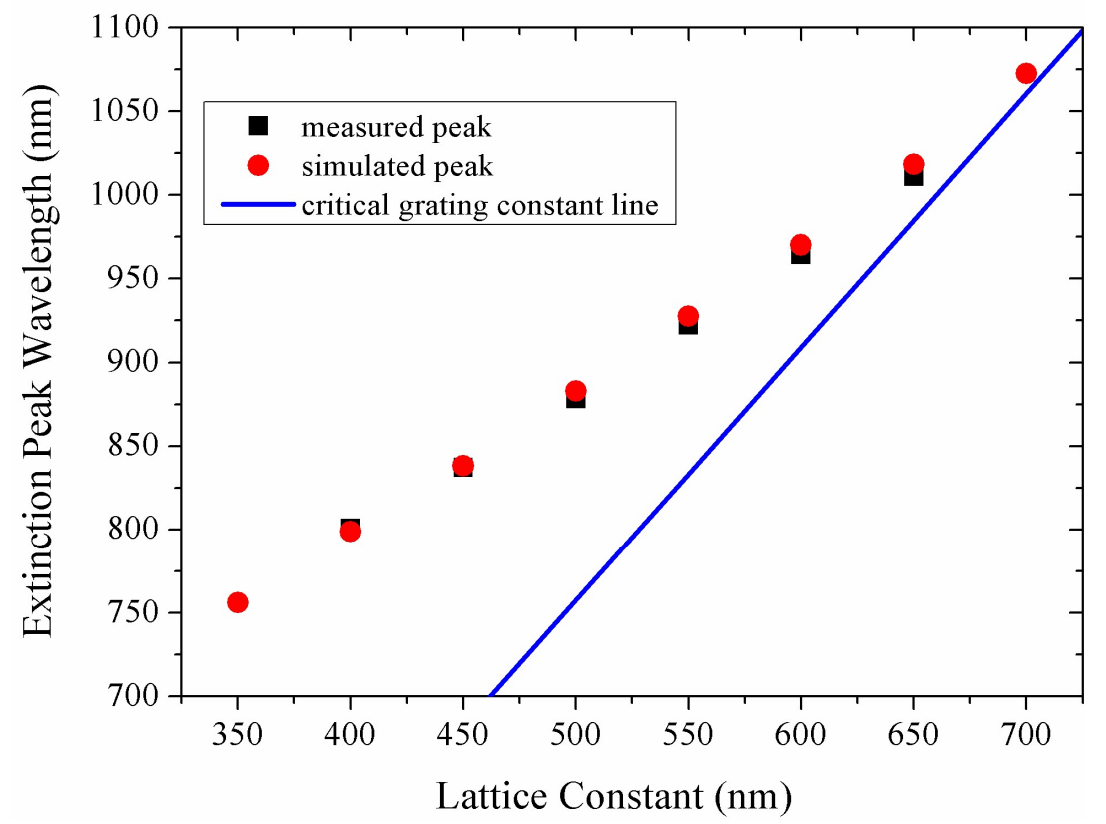

\section{FIG.5b}

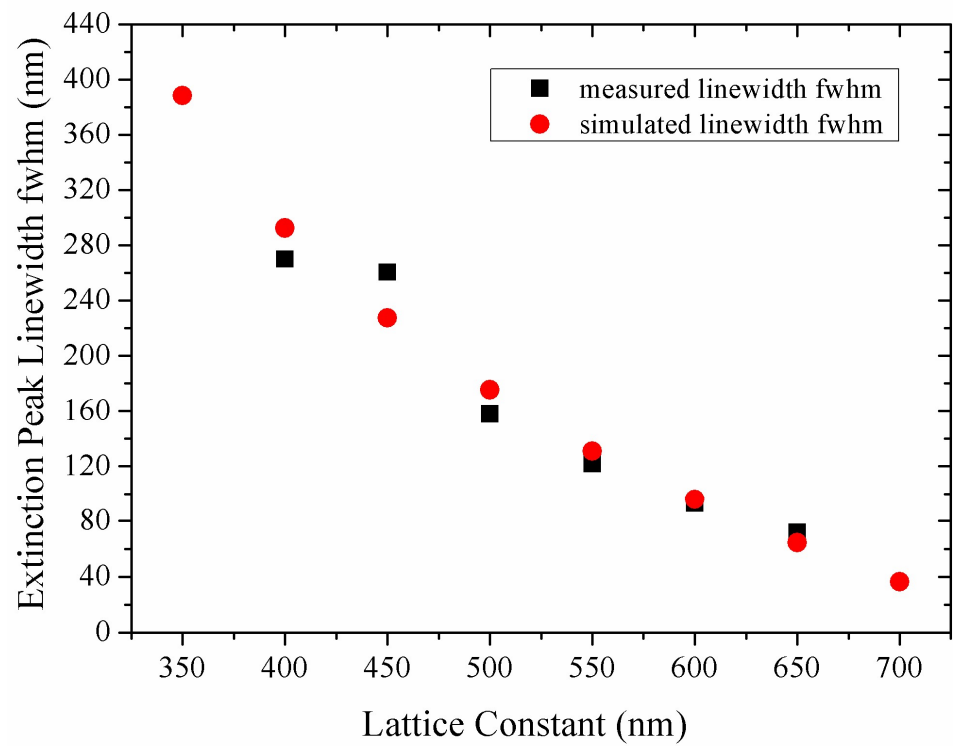


Fig.5c

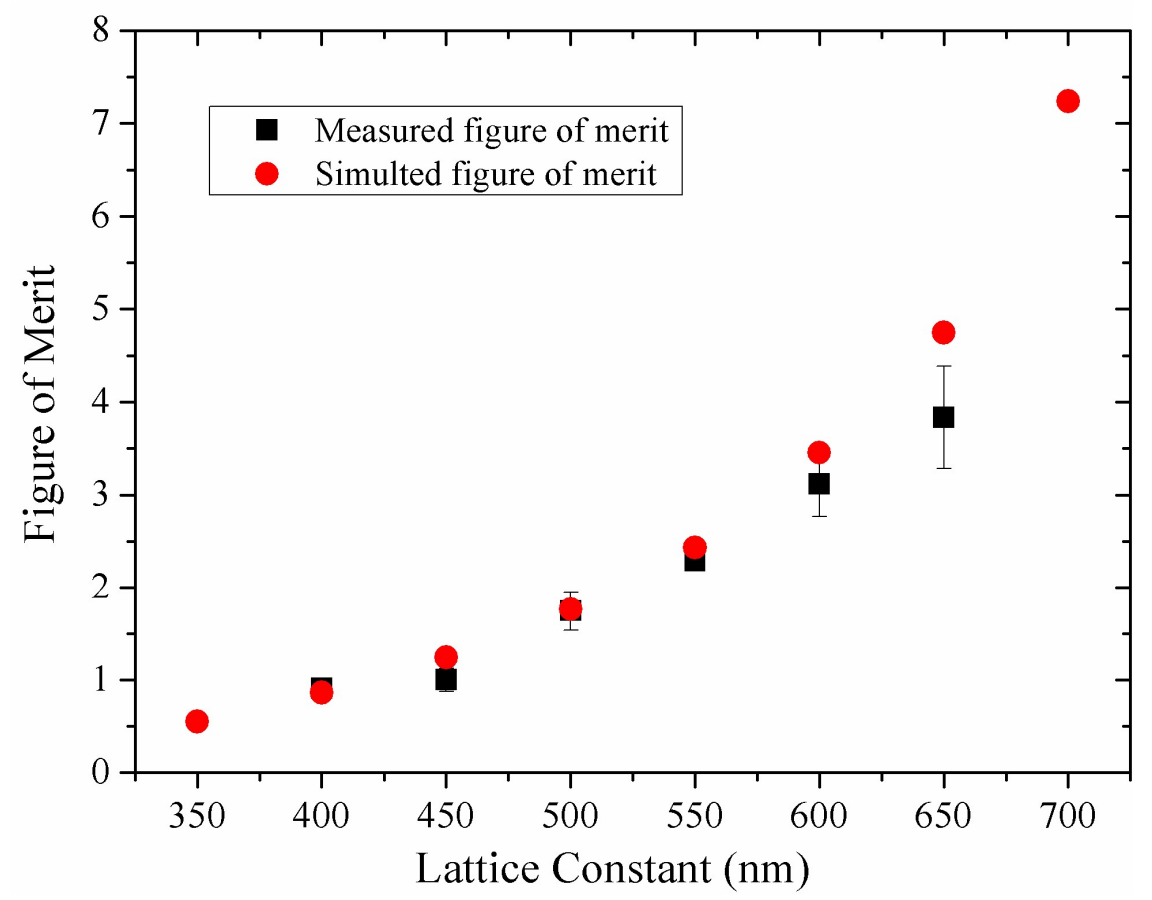

FIG.6

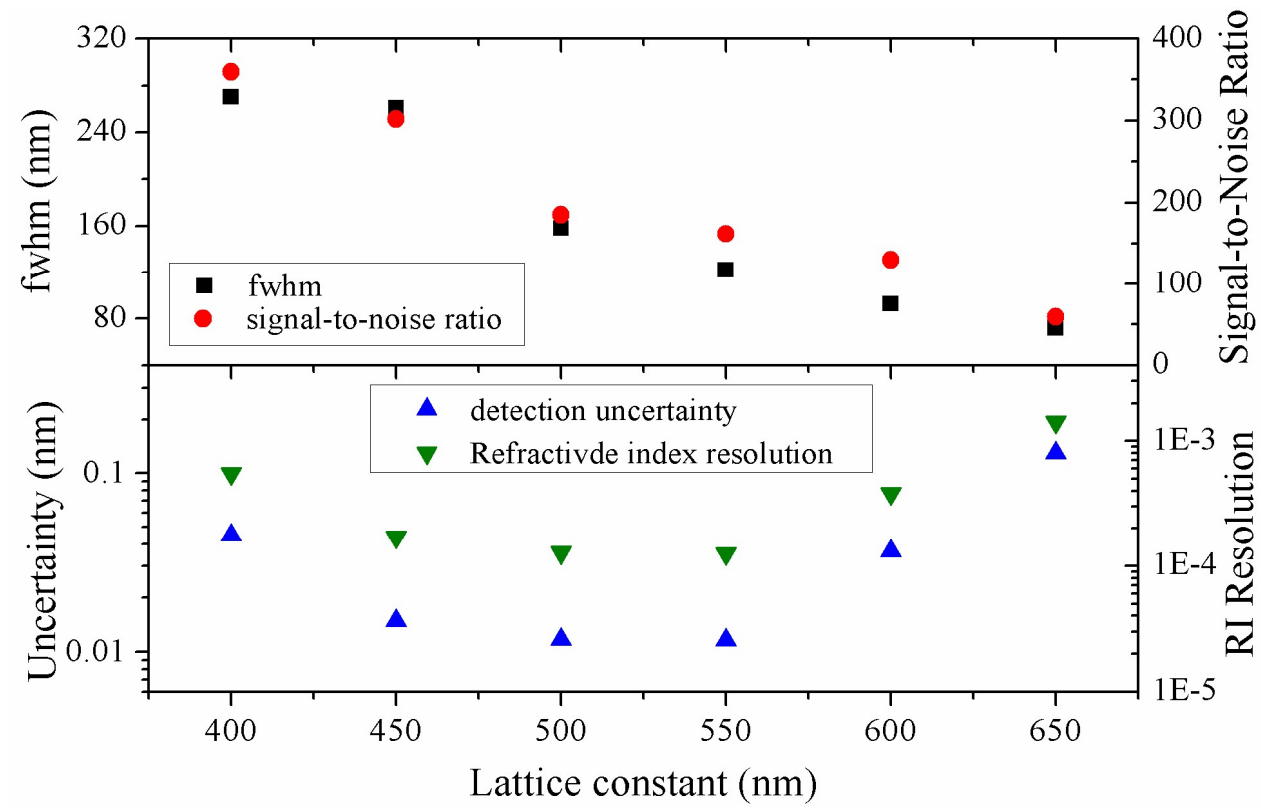


FIG.7

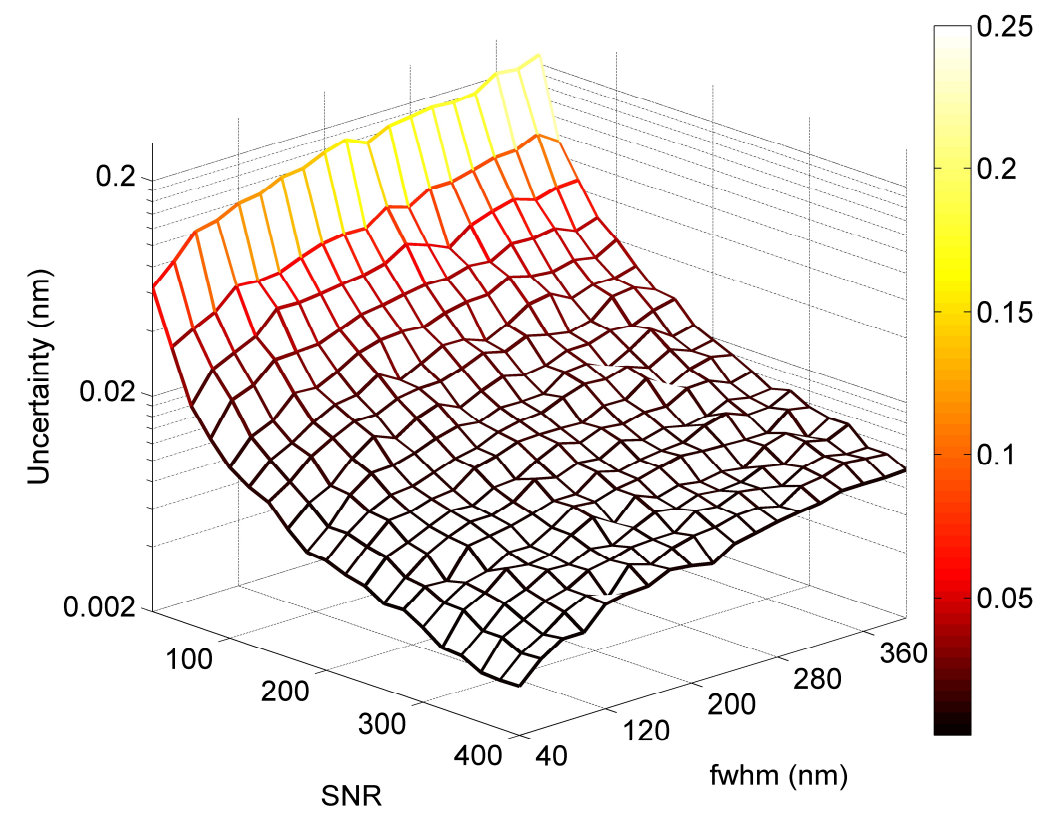

FIG.8a

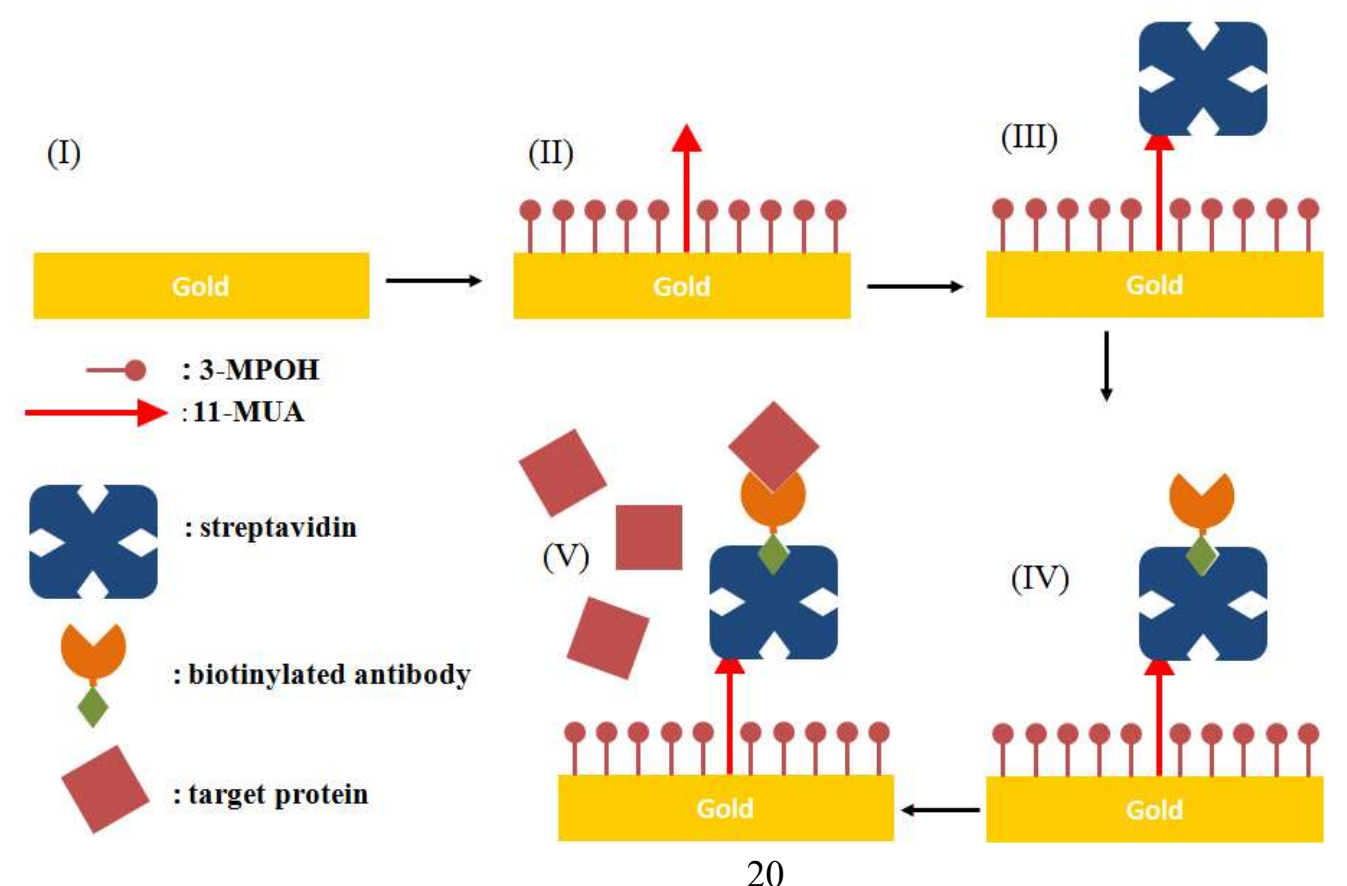




\section{FIG.8b}

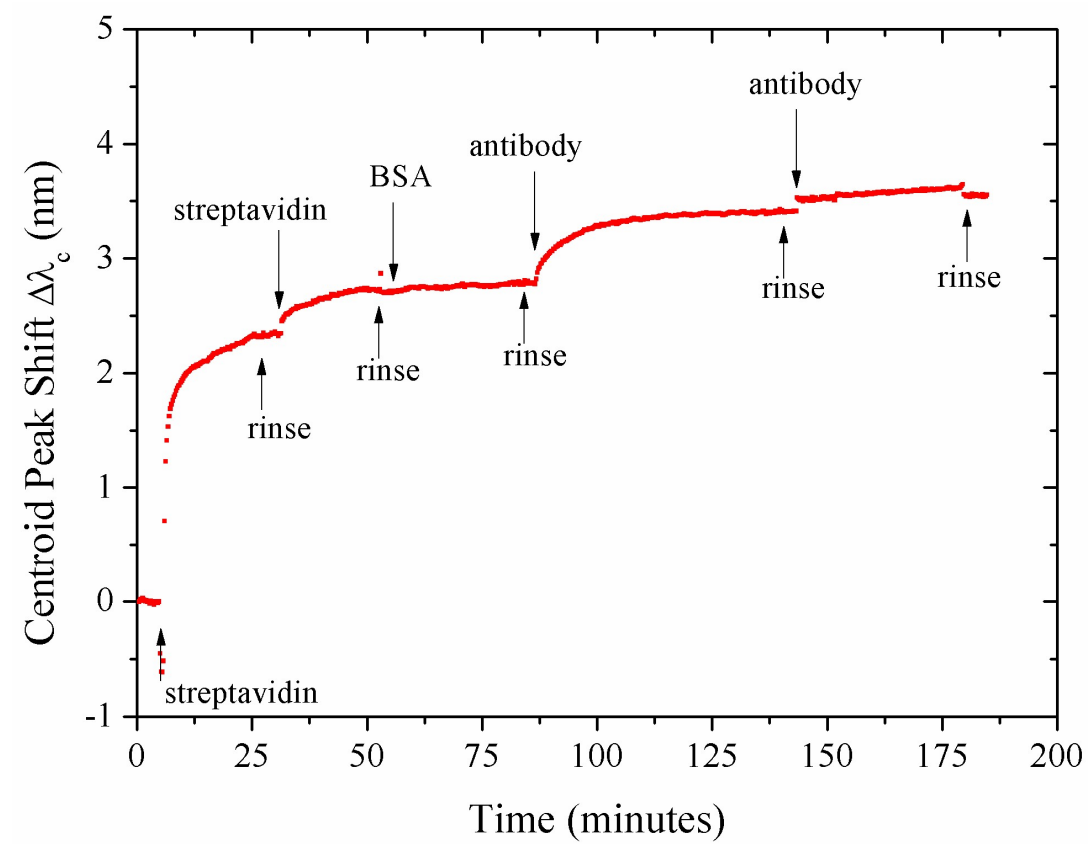

FIG.9

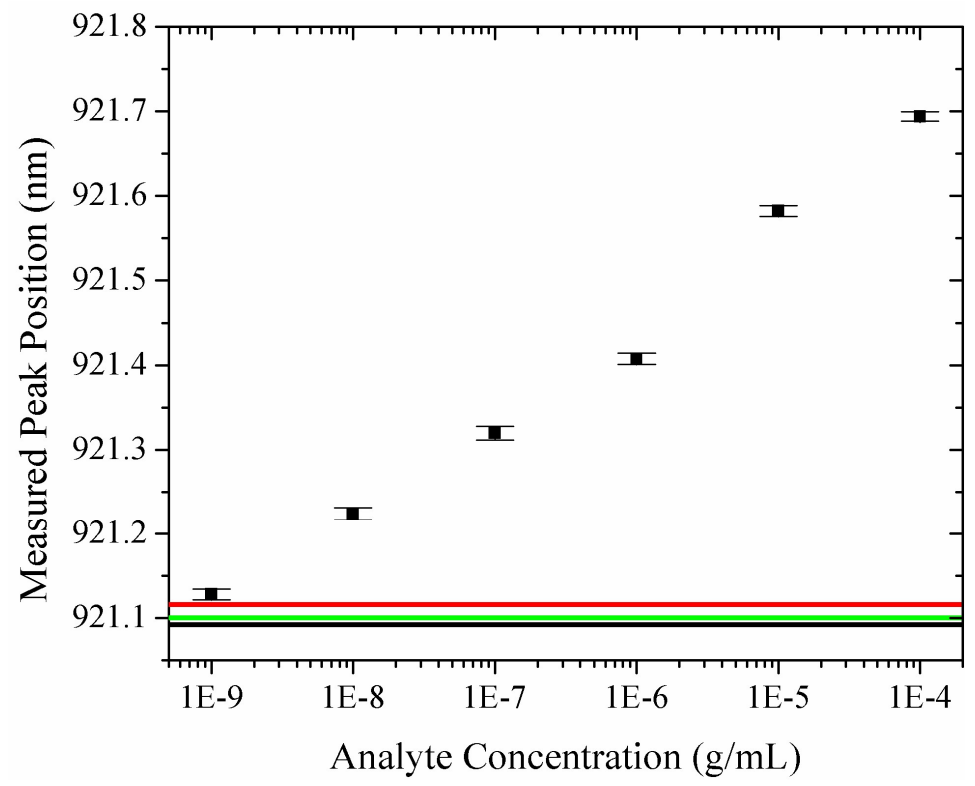

